\title{
Electron-hadron shower discrimination in a liquid argon time projection chamber
}

\author{
J.J. Back, G.J. Barker, A.J. Bennieston, S.B. Boyd, B. Morgan, Y.A. Ramachers ${ }^{\text {a }}$ \\ Dept. of Physics, University of Warwick, Coventry CV4 7AL, UK
}

Received: 26 October 2012 / Revised: 18 February 2013 / Published online: 21 March 2013

(C) The Author(s) 2013. This article is published with open access at Springerlink.com

\begin{abstract}
By exploiting structural differences between electromagnetic and hadronic showers in a multivariate analysis we present an efficient Electron-Hadron discrimination algorithm for liquid argon time projection chambers, validated using Geant4 simulated data.
\end{abstract}

\section{Introduction}

Liquid Argon Time Projection Chambers (LAr-TPCs) are recognised as being a potential detector technology for a next-generation Neutrino oscillation experiment and their development is the subject of vibrant R\&D programmes in Europe, Japan and the USA (see Refs. [1-3] and references therein). The simultaneous tracking and calorimetry capability, with millimetric granularity, makes LAr-TPCs ideal to image Neutrino interactions over a wide range of energies as demonstrated recently by ICARUS [4].

Despite the physics promise of LAr-TPCs, a fully automated (that is, with zero human interaction) software system for reconstructing events has proven difficult to achieve. With the availability of more and more event data, recent years have seen progress in limited clustering of structures and tracking. However, a general event is likely to contain a mixture of showers and tracks originating from an unknown vertex anywhere in the sensitive volume. One major challenge for automated reconstruction is to disentangle the fundamentally different shower and track structures and hence segment the event into its final state particles. Accurate segmentation is critical for subsequent particle identification on the substructures. Neutrino oscillation analyses proposed for next-generation facilities rely heavily on accurate interaction classification, motivating studies of this task.

In this article, we concentrate on the particle identification step, based on extensive Monte-Carlo simulations, as-

a e-mail: y.a.ramachers@warwick.ac.uk suming exact segmentation, i.e. all hits belonging to each single particle have been clustered with full efficiency and purity. We defer discussion of techniques for this more challenging task to later publications. We present a study using a set of effective and computationally simple variables which allow the discrimination of Electron shower structures from all relevant hadronic shower structures in a finegrained tracking detector such as a LAr-TPC. For the intended physics application, the dominant Hadron shower structures result from Pions, Protons or Kaons in the final state. In this context, Electron-Hadron shower discrimination is important for Neutrino oscillation studies in order to measure Electron-neutrino events in a Muon-neutrino beam, resulting in an energetic Electron in the LAr medium after a charged-current interaction with a nucleus. Therefore, the Electron is considered to be the "signal" and anything nonelectron, i.e. Hadron showers, is labelled as "background", unless stated otherwise.

Additionally, the energy resolution of any LAr-TPC will depend on the nature of the particle depositing energy due to quenching factors, i.e. particle dependent ionization efficiencies in liquid argon. Electromagnetic and hadronic particle energy depositions will each require separate energy calibrations depending on their specific ionization as a function of energy, hence for their energy measurement particle identification would be beneficial.

The set of variables also prove to be useful for other particle identification tasks. Track structures associated with Protons or Muons can be identified by these variables as well as, to a lesser extent, hadronic final states such as neutral and charged Pions and Kaons.

In the following, we describe the Monte-Carlo data production in order to define our case studies, then the analysis algorithm by first introducing the discrimination variables before finally presenting their applicability to identifying particles in various Neutrino interaction event topologies. 


\section{Monte-Carlo data production}

Several simulated single particle event classes were prepared as input to the analysis. We have tested the algorithm with a Muon-neutrino flux generated as part of the LAGUNA-LBNO FP7 programme [5, 6] to evaluate the physics potential of a long baseline experiment between CERN and an underground far detector location in Pyhasalmi Finland (CN2PY). The CN2PY Neutrino flux [7] (un-oscillated) from decaying negatively charged Pions (for a distance of $2300 \mathrm{~km}$ ) provided the input for the first case

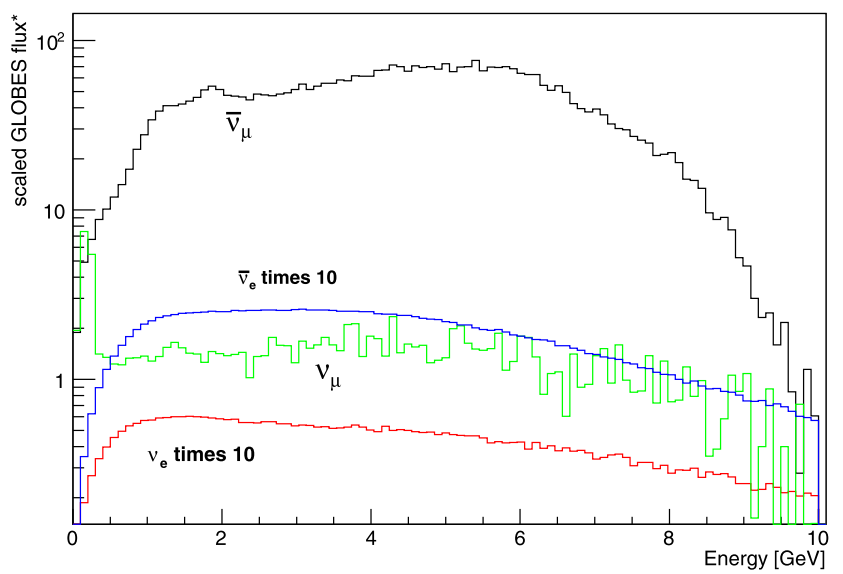

Fig. 1 The scaled CN2PY Neutrino beam fluxes [7] from negative Pion decays. Weighting factors for the fluxes are given in Table 1. *: Flux units are prepared for use in GLoBES [15] and scaled here by a factor $10^{-12}$. Parameters entering the calculation of the correct GLoBES normalisation factor in order to obtain SI flux units are: $3 \mathrm{e} 21$ p.o.t., a distance of $100 \mathrm{~km}$ and an area of $100 \mathrm{~m}^{2}$ with a detector at a distance of $2300 \mathrm{~km}$. For details, see the GLoBES user manual

Table 1 The relative neutrino flavour weighting factors for the CN2PY Neutrino beam (un-oscillated) from negatively charged Pions taken as input for this study. Note that the uniform Neutrino energy beam case study also takes these flavour weighting factors into account

\begin{tabular}{llll}
\hline $\bar{v}_{\mu}$ flux & $v_{\mu}$ flux & $\bar{v}_{e}$ flux & $v_{e}$ flux \\
\hline 1.0 & $3.471 \times 10^{-2}$ & $4.455 \times 10^{-3}$ & $1.0772 \times 10^{-3}$ \\
\hline
\end{tabular}

Table 2 The relative weighting factors for the final state particles resulting from the CN2PY Neutrino beam simulation containing particles given in the first column. These weighting factors are each taken into account during the multivariate analysis stage and calculated by multiplying relative simulated final state particle numbers with the cor- study. Neutrino reaction final states were produced using the GENIE event generator (version 2.6.4) [8]. The scaled Neutrino energy distributions are shown in Fig. 1 with relative weighting factors given in Table 1.

The GENIE simulated events were un-filtered, with all possible final state particles allowed. The total number of each individual particle final state from GENIE in each of the four Neutrino fluxes then results in individual weighting factors for that particle species during the subsequent analysis. The complete set of weighting factors used during the multivariate analysis, see below, is listed in Tables 2 and 3.

These single particle energy distributions from the GENIE simulations then serve as the particle source in the Geant4 (version 9.5.0) Monte-Carlo transport simulation toolkit [9]. The simulation models an homogeneous liquid argon detector as a cylinder with a radius and height of $100 \mathrm{~m}$, in order to fully contain the showers. Particles start at the centre of the cylindrical volume and are tracked through the volume with interactions modelled by the QGSP_BIC_HP physics list, with all electromagnetic and hadronic processes enabled, as recommended by the Geant 4 collaboration for our energy range of interest. The LAr volume is divided into $(1 \times 1 \times 1) \mathrm{mm}^{3}$ cubic voxels, and all primary and secondary particles are tracked through voxels down to zero energy, or until they leave the volume. Energy deposits by charged particles passing through voxels are tallied into a map between voxel coordinates and the total energy deposited. No attempt was made to model the readout system, but quenching of ionisation events specific to LAr detectors was taken into account following [10].

Results are collected and processed in a final step by four selected multivariate analysis algorithms (MVA) taken from the TMVA toolkit [11]. Each MVA is based on supervised learning, requiring separate training and test data samples. Each sample is chosen from a random split in half of the available data. The final evaluation delivers optimal cuts for each selected MVA method, producing efficiencies for signal selection, background selection and corresponding purities. Note that the complete set of weighting factors as listed in Tables 2 and 3 is used in each MVA method. The

\begin{tabular}{llllllll}
\hline & $\pi^{+}$ & $\pi^{-}$ & $\pi^{0}$ & $p$ & $K^{ \pm}$ & $K^{0}$ & $\gamma$ \\
\hline $\bar{v}_{\mu}$ & 0.203 & 0.508 & 0.409 & 1.610 & 0.016 & 0.022 \\
$v_{\mu}$ & $2.42 \times 10^{-2}$ & $9.89 \times 10^{-3}$ & $2.04 \times 10^{-2}$ & $6.284 \times 10^{-1}$ & $1.43 \times 10^{-3}$ & $1.1 \times 10^{-3}$ & $1.96 \times 10^{-3}$ \\
$\bar{v}_{e}$ & $9.4 \times 10^{-4}$ & $2.32 \times 10^{-3}$ & $1.85 \times 10^{-3}$ & $7.2 \times 10^{-3}$ & $7.7 \times 10^{-5}$ & $1.1 \times 10^{-4}$ & $1.8 \times 10^{-4}$ \\
$v_{e}$ & $7.6 \times 10^{-4}$ & $3.1 \times 10^{-4}$ & $6.4 \times 10^{-4}$ & $1.95 \times 10^{-3}$ & $4.5 \times 10^{-5}$ & $3.4 \times 10^{-5}$ & $5.7 \times 10^{-5}$ \\
\hline
\end{tabular}

responding neutrino flavour weighting factors (see the discrepancy on magnitude between $\bar{v}_{\mu}$ values and all other weighting factors due to the small neutrino flavour weighting factors). Note that Muon and Electron energy spectra are taken from Genie simulations, also using the corresponding neutrino flavour weighting factor 
Table 3 Same as Table 2 but for the second case study of a uniform neutrino energy beam

\begin{tabular}{llllllll}
\hline & $\pi^{+}$ & $\pi^{-}$ & $\pi^{0}$ & $p$ & $K^{ \pm}$ & $K^{0}$ & $\gamma$ \\
\hline $\bar{v}_{\mu}$ & 0.251 & 0.567 & 0.466 & 1.635 & 0.023 & 0.029 \\
$v_{\mu}$ & $2.61 \times 10^{-2}$ & $1.13 \times 10^{-2}$ & $2.25 \times 10^{-2}$ & $6.32 \times 10^{-2}$ & $1.68 \times 10^{-3}$ & $1.28 \times 10^{-3}$ & $2.13 \times 10^{-3}$ \\
$\bar{v}_{e}$ & $1.1 \times 10^{-3}$ & $2.54 \times 10^{-3}$ & $2.1 \times 10^{-3}$ & $7.29 \times 10^{-3}$ & $9.9 \times 10^{-5}$ & $1.4 \times 10^{-4}$ & $1.9 \times 10^{-4}$ \\
$\nu_{e}$ & $8.1 \times 10^{-4}$ & $3.5 \times 10^{-4}$ & $7.1 \times 10^{-4}$ & $1.97 \times 10^{-3}$ & $5.4 \times 10^{-5}$ & $3.9 \times 10^{-5}$ & $6.7 \times 10^{-5}$ \\
\hline
\end{tabular}

chosen methods are: the k-nearest neighbour search (KNN), two variations of boosted decision trees (BDT, BDTG) and a neural network (MLPBNN). These four represent the most successful out of a substantially larger number of methods in the TMVA toolkit during preliminary tests on our project.

Our second case study attempts a pure algorithm test by running the identical analysis as discussed above over a sample of final state particles sampled from a uniform Neutrino energy beam (same flavour weighting factors, same energy range as the $\mathrm{CN} 2 \mathrm{PY}$ case). This case studies the impact of a less well constrained neutrino beam energy distribution.

\section{Electron-shower discrimination algorithm}

Below, we detail the calculation and physics motivation of four discriminating variables. The main assumption underpinning all of the following is that clustered energy deposits, hits, in the detector from a particular particle have been collected as a single object by the reconstruction, i.e. a cluster. Results are collected and processed in a final step by four selected multivariate analysis algorithms (MVA) taken from the TMVA toolkit [11]. This final step is discussed in more detail in Sect. 2.

This crucial clustering step for any LAr detector analysis is assumed to have taken place already. It should be stressed that a reliable, complete and automatic initial clustering step is, in fact, among the unsolved challenges of LAr data analysis, owing to the richness of topological event structures in such a detector, and will not be addressed here.

The first step of the discrimination algorithm consists of transforming the isolated cluster using a principal component analysis (PCA) [12]. The data at this stage consists purely of detector hits forming a point-cloud of unknown shape. Dealing with LAr detectors translates into the capability of assigning three spatial coordinates and a charge value to each individual hit (with corresponding uncertainties).

The reason for the initial PCA step is that it allows us to consider the unknown point-cloud to be aligned and ordered in a consistent way. The PCA is set up such that it calculates the three principal spatial components (ignoring the charge of the hit) as eigenvalues of the transformation matrix, which itself consists of the corresponding eigenvectors. Applying the transformation aligns the coordinate axes to the principal axes, using the convention that the dominant principle axis becomes the ' $x$ '-axis in a right-handed Cartesian coordinate system. This allows to utilise transverse and longitudinal geometric information due to the presence of a well defined axis for the structure.

\subsection{The lateral projection variable "lat"}

This discrimination variable is motivated by previous and ongoing accelerator experiments and their analysis of electromagnetic (EM) and Pion showers (see for example Ref. [13]). The entire point-cloud is projected onto the plane of minor principal components (the lateral plane to the major principal axis). A core region is defined around the projected mean value and the ratio of energy (charge) deposited in the core to the total energy (charge) yields the "lat" variable.

Charged Hadron showers feature either a dense core region, often resembling a track-like structure with only a thin "halo" around it, or dissolve into a wide-spread, loose cloud of hits. Proton tracks might or might not shower at all. EM showers, by contrast, tend to produce more constant halo-tocore ratios, as shown in Figs. 2, 3 and 4.

The precise definition of the core region turns out to be non-critical. For simplicity, a circular shape is chosen, which is centred on the mean value of the projected point-cloud and is defined with half the Molière radius in liquid argon, which is equal to $9.61 \mathrm{~cm} \mathrm{[14].} \mathrm{Each} \mathrm{hit} \mathrm{inside} \mathrm{the} \mathrm{core} \mathrm{contributes}$ to the sum of energy in the core, which is then divided by the total energy of the shower (the sum of the energies of all hits).

\subsection{The hit concentration variable "con"}

While the previous variable emphasises a subdivision of the shower structure into two main components (core and halo), the hit concentration variable "con" attempts to use the same lateral information globally. This is independent of any material constant, such as the Molière radius.

The variable first calculates the radial distance of each hit from the main principal axis, i.e. the radial distance from the origin in the lateral projection plane. A minimum threshold radius is set in order to avoid the Coulomb singularity (for 
Fig. 2 The set of four discrimination variables applied to the CN2PY beam (see text) Neutrino events. Signal events are defined as electromagnetic shower events from Electrons, compared to the complete shower background, which includes all simulated structures from Protons, charged Pions, neutral Pions, charged and neutral Kaons and Muons, all with appropriate weighting factors. For MVA training purposes the event sample size is set to $10^{4}$ for each individual signal and background contribution. The histograms for signal and background are scaled individually to equal integrals for display purposes only

Fig. 3 Similar to Fig. 2; the set of four discrimination variables but for the second case study of a uniform neutrino energy beam
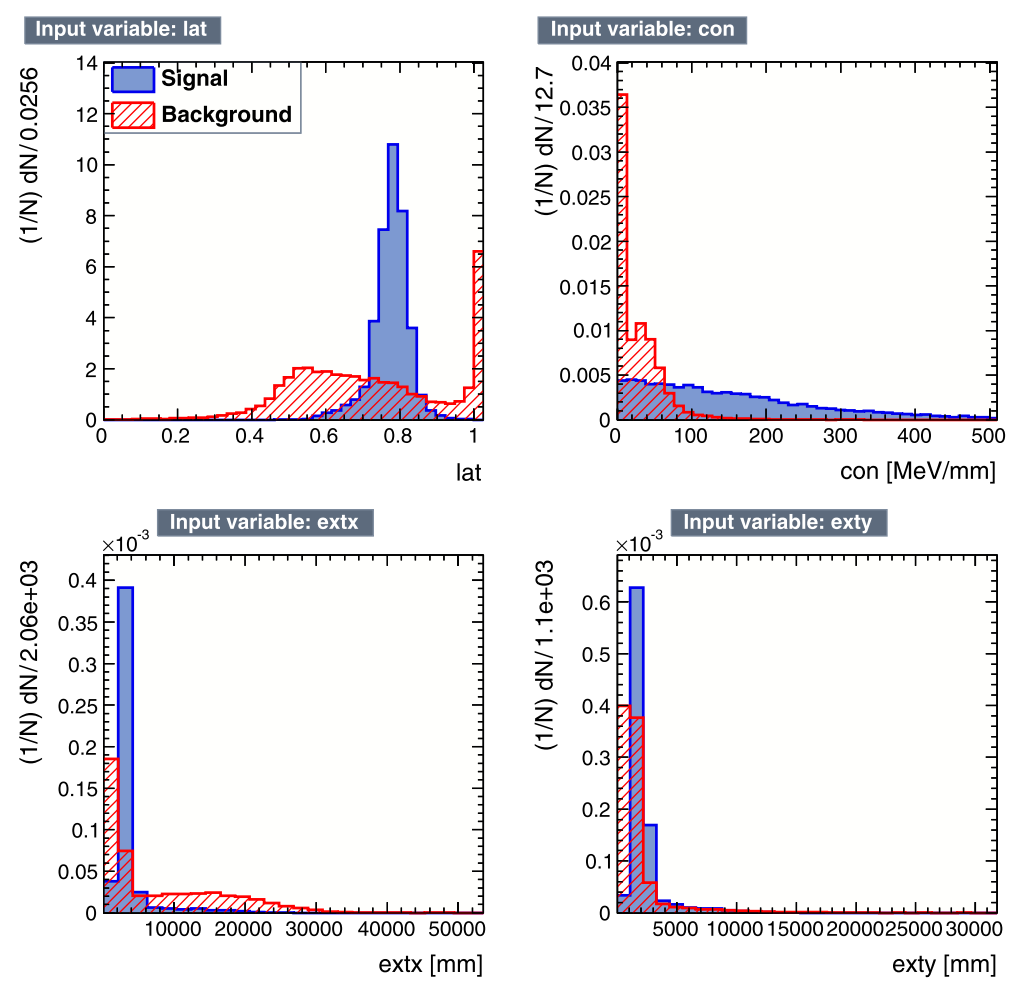
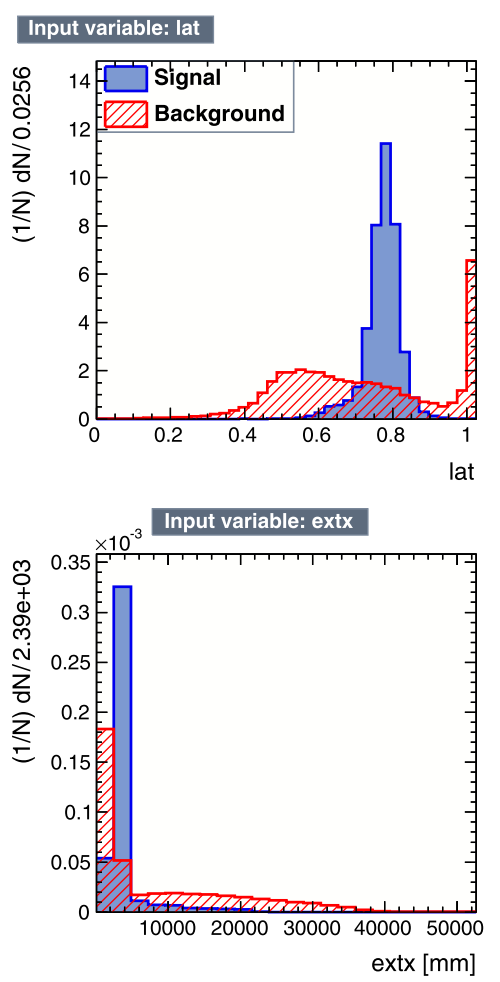

\section{Input variable: con}
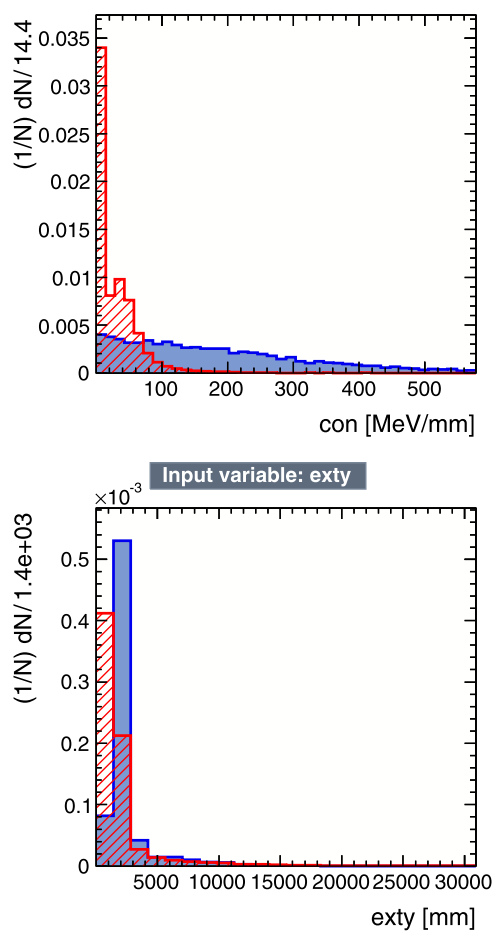

practical purposes chosen as $1 \mathrm{~mm}$ in this analysis), and finally the hit concentration is calculated as the sum of charge (energy) of the hits divided by their radial distance.
The idea is to calculate the Coulomb potential energy of the point-cloud around the main principal axis. This approach delivers complementary information to the previ- 


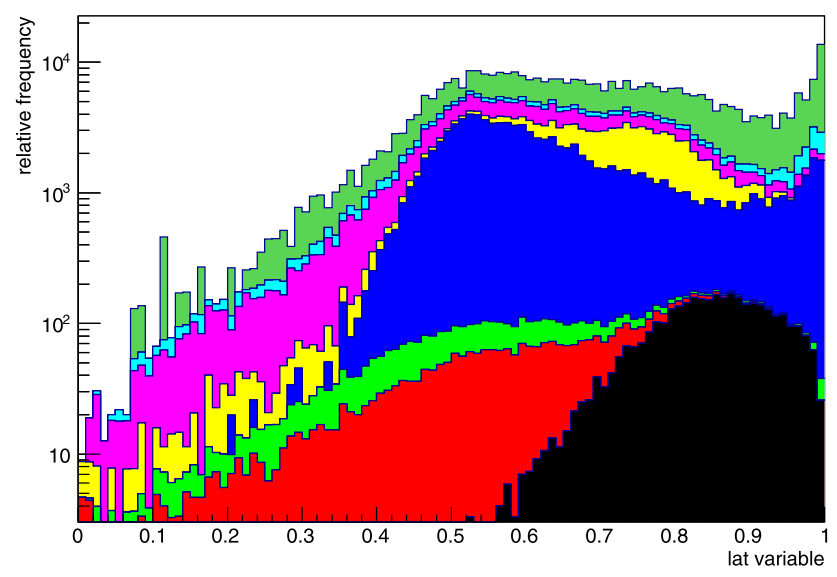

Fig. 4 Stacked histogram example displaying the background composition due to simulated final state particles from the CN2PY neutrino beam case. This example shows the background in the 'lat' variable (left panel) for an electron signal for the dominant anti-muon neutrino flavour and the 'con' variable on the right panel. The individual histograms are normalised and scaled subsequently by their cor-

ous rigid division into two parts, since it emphasises the extremes such as track-like structures as being particularly highly concentrated, and very loose structures as being particularly low in concentration. Figures 2, 3 and 4 show the main benefit of this variable. The Hadron showers are dominated by less densely concentrated structures (they peak at low Coulomb energies around the main principal axis). A second population of Hadron events contain Proton tracks as well as sufficiently dense Pion tracks, contributing a larger value to this concentration variable. EM showers are rather non-specific but are significantly more concentrated than Hadron showers.

\subsection{Spatial extent variables "extx, y"}

The final two proposed discrimination variables are obtained by quantifying the spatial extent of the hit cloud in the three available principal component axes. A definition of the extent in purely geometrical terms turns out to be very useful. The algorithm calculates extent in all three available axes but one of the two transverse axes is discarded since it does not contribute any new information. This azimuthal symmetry assumption around the dominant principal axis was extensively tested and confirmed during this study.

The spatial extent is calculated by obtaining the convex hull in 3D around the hit cloud and finding the maximum distance (extent) of the hull on all three axes. Building the convex hull around an object is a standard algorithm in image analysis [16]. Even though its application in 3D is rather exotic, ready-made implementations of the code exist, for instance in the scientific python library, SciPy [17]. The meaning of the term convex hull can be visualised by

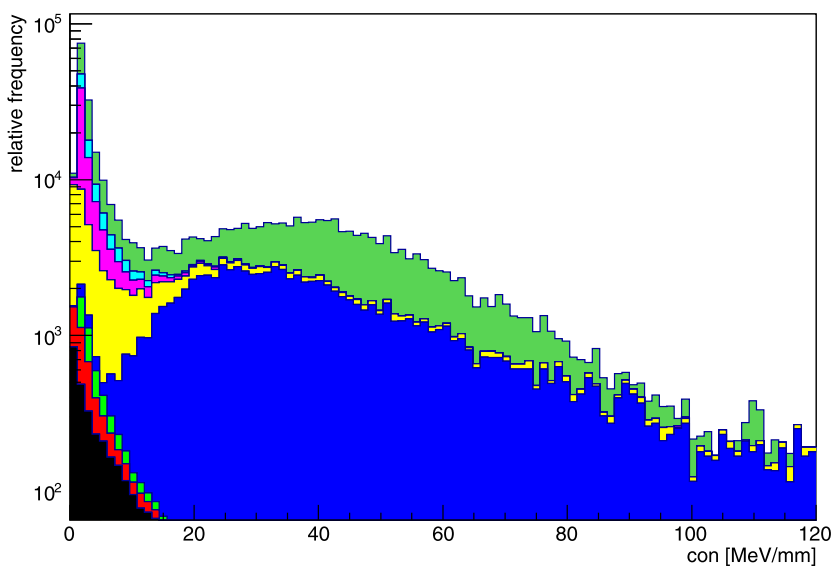

responding weighting factor (particle dependent) from Table 2. The additional neutrino flavours contributing to that background in Fig. 1 are suppressed to avoid clutter from too many colours on the plot. The colour coding is as follows: black-gamma, red- $K^{0}$, greencharged Kaons, blue-Muon, yellow-neutral Pion, pink- $\pi^{-}$, light blue $-\pi^{+}$, dark green-Proton (Color figure online)

considering stiff packaging paper, wrapped around an irregularly shaped object, i.e. the hit cloud, in order to enclose it completely. The paper would represent the convex hull, without the folds and overlaps real paper would produce.

The convex hull is obtained by calculating the Delaunay tessellation [16] of the hit cloud and finding the outermost Delaunay triangles, made of data members, enveloping the entire cloud. The difference between the maximum and minimum coordinate value on each (principal component) coordinate axis is calculated, giving the extent of the hull in all three dimensions.

\section{Results}

4.1 Electron to combined hadron/lepton background discrimination

Results in this section summarise the performance of the proposed discriminating variables when attempting to identify Electrons in a data sample consisting of all dominant final state particles, i.e. charged and neutral Pion, Protons, Kaons and Muons. The most straightforward way of illustrating the effectiveness of the proposed variables can be seen in Fig. 5, showing the background rejection as a function of signal efficiency for all four chosen methods, the nearest neighbour (KNN), two variations of boosted decision trees (BDT, BDTG) and a neural network (MLPBNN). These plots would ideally show a complete background rejection for all signal efficiencies up to $100 \%$. The closer the curves follow these ideal lines on the figure, the better the discrimination of the selected MVA method. The MVA 


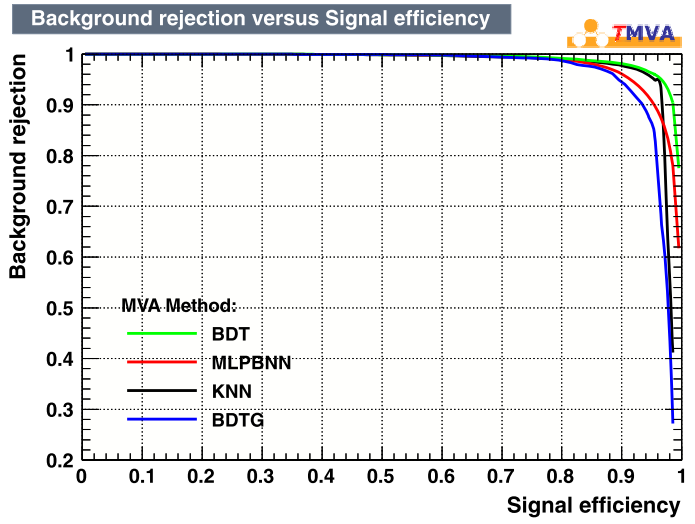

Fig. 5 Background rejection as a function of the signal efficiency for all four chosen methods for an EM shower signal in a (left panel) $\mathrm{CN} 2 \mathrm{PY}$ beam and (right panel) uniform neutrino energy distribution. The methods are: the k-nearest neighbour search (KNN), two varia-

Table 4 Particle identification efficiencies and contamination (background efficiency or 'contamination' is equal to 1.0-background rejection) on electron shower detection in a combined background of Hadron showers and Muons for all selected MVA methods. Weight-

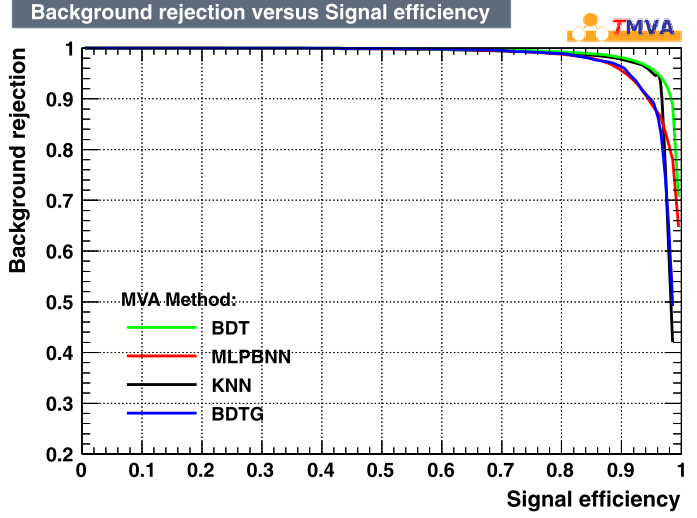

tions of boosted decision trees (BDT, BDTG) and a neural network (MLPBNN), all originating from the TMVA toolkit [11]. For numerical results on this analysis, see Tables 4 and 5

ing factors for each final state particle are always taken into account for signal as well as background particles. Efficiencies are calculated for the optimal decision boundary values defined by maximising the signal-to-total event ratio, $S / \sqrt{(S+B)}$

\begin{tabular}{llll}
\hline MVA method & Beam case study & Electron-signal/background efficiency [\%] & Signal-to-total $S / \sqrt{(S+B)}$ \\
\hline KNN & CN2PY & $95.7 / 5.2$ & 30.1 \\
MLPBNN & CN2PY & $93.9 / 7.4$ & 29.5 \\
BDT & CN2PY & $96.2 / 4.5$ & 30.3 \\
BDTG & CN2PY & $90.6 / 6.0$ & 29.2 \\
KNN & Uniform v & $95.4 / 5.0$ & 30.1 \\
MLPBNN & Uniform v & $92.9 / 7.3$ & 29.4 \\
BDT & Uniform v & $96.1 / 4.9$ & 30.2 \\
BDTG & Uniform $v$ & $90.6 / 4.2$ & 29.4 \\
\hline
\end{tabular}

methods mentioned above have been chosen as the most effective among a list of 24 possible methods offered in the TMVA toolkit.

Quantitative results for efficiencies and purities in EM shower discrimination are given in Table 4, and illustrate a successful (well above $90 \%$ signal efficiency and background rejection) analysis technique in distinguishing EM showers from Hadron showers in a LAr detector at all interesting Neutrino energies. Finally, the method appears relatively insensitive to presence or absence of a priori beam knowledge.

\subsection{Additional applications}

The variables discussed above lend themselves to further applications simply because they are sensitive to only the geometrical shapes of clouds of hits in 3D space as opposed to kinematic variables. As a first application we consider neutral current (NC) interactions in LAr. NC interactions form an important background to Neutrino oscillation signals since they can produce neutral pions in an event. Due to their prompt decay into a pair of photons, with the inevitable EM showers, they constitute a problematic background to Electron-neutrino charged-current interactions. This means that identifying final state $\pi^{0}$ would be beneficial for several reconstruction tasks. One study [18] delivered a strong discrimination factor of $10^{-3}$ for mis-identified $\pi^{0}$ in a LArTPC, a number which is widely quoted. Our efforts here deliver a weaker $\pi^{0}$ discrimination, see below. However it does not rely on complex computational tasks such as shower separation and fitting and subsequent vertex finding in a potentially very busy event structure, i.e. the reason why [18] relies on visual event selection.

The optimal signal and background efficiencies for the $\pi^{0}$ selection are shown in Table 5 (see also Fig. 6). Just as for the study on Electron to Hadron discrimination, all subsequent studies on changing the signal, here to the $\pi^{0}$ particle, consider the complete set of background particles and 
Fig. 6 Similar to Fig. 2 for the CN2PY beam producing a $\pi^{0}$

final state particle signal

compared to the complete

shower background

\section{Input variable: lat}
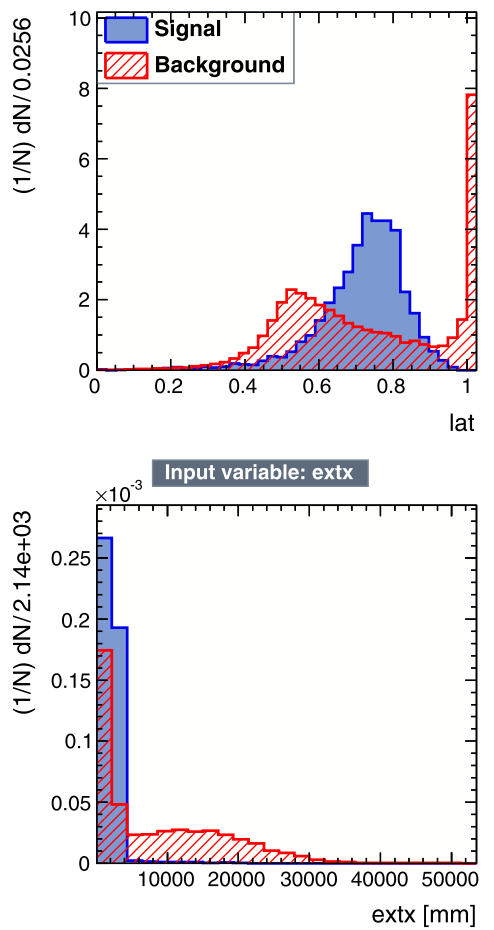
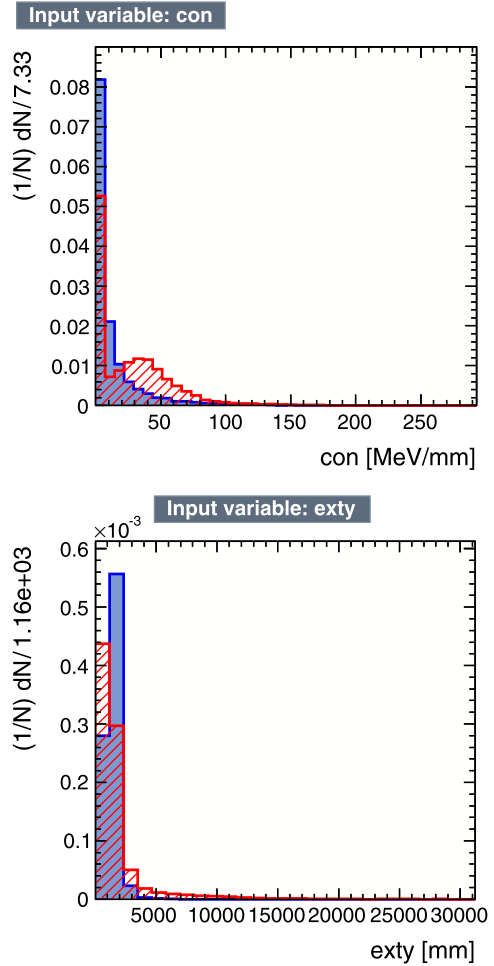

Table 5 Particle identification efficiencies and contamination (background efficiency or 'contamination' is equal to 1.0-background rejection) in a combined background of Hadron showers and Leptons for the best MVA method, boosted decision trees, BDT. Efficiencies are calculated for the optimal decision boundary values defined by maximising the signal-to-total event ratio, $S / \sqrt{(S+B)}$. Signal particles are listed in the top row

\begin{tabular}{|c|c|c|c|c|c|c|}
\hline \multirow[t]{2}{*}{ MVA method } & \multirow[t]{2}{*}{ Beam case study } & \multicolumn{5}{|c|}{ Particle signal/background efficiency [\%] } \\
\hline & & $\pi^{0}$ & Muon & Proton & Kaon & $\pi^{+}$ \\
\hline BDT & CN2PY & $96.8 / 15.9$ & $97.6 / 1.4$ & $93.7 / 28.3$ & $93.4 / 24.0$ & $97.3 / 41.7$ \\
\hline BDT & Uniform $v$ & $96.1 / 15.6$ & $98.1 / 2.3$ & $92.8 / 29.9$ & $92.2 / 25.1$ & $97.9 / 43.1$ \\
\hline
\end{tabular}

weighting factors, listed in Table 2 and additionally the relevant leptons, i.e. Muons and Electrons. All final state particles other than the selected signal particle constitute background.

Other final state particles can also be identified as signals with varying degrees of success. In Table 5 we list results for Muons (see Fig. 7), Protons, Kaons and $\pi^{+}$, where $\pi^{-}$ results are practically identical to Kaon results, hence have been left out.

\subsection{Detector threshold effects}

Detector-specific effects depend heavily on our primary assumption of successful clustering of hits belonging to a single particle in an overall unspecified detector event. However, any automated analysis will at some point have to solve this challenge for finely-grained LAr detector devices.
Nevertheless, threshold variations would remove (or add) hits to the total simulated single particle event and potentially change geometrical structures. In order to test the robustness of our method against such a realistic detector effect, we studied two scenarios: one realistic hit energy cut at $120 \mathrm{keV} / \mathrm{mm}$ for quenched (measured) energies and one essentially without an hit energy cut $(1 \mathrm{keV} / \mathrm{mm})$. The threshold choice of $120 \mathrm{keV} / \mathrm{mm}$ originates from the simulated onset of the Muon energy loss peak which would be left practically fully intact by this cut. The minimal cut scenario at $1 \mathrm{keV} / \mathrm{mm}$ roughly doubles the total amount of hits for most single particles simulated.

The change from these threshold effects on all of the particle identification results shown earlier is negligible, typically less than $0.3 \%$ on any signal or background efficiency quantity. 
Fig. 7 Similar to Fig. 2 for the CN2PY beam producing Muons in the final state, here defined as signal compared to the complete shower background
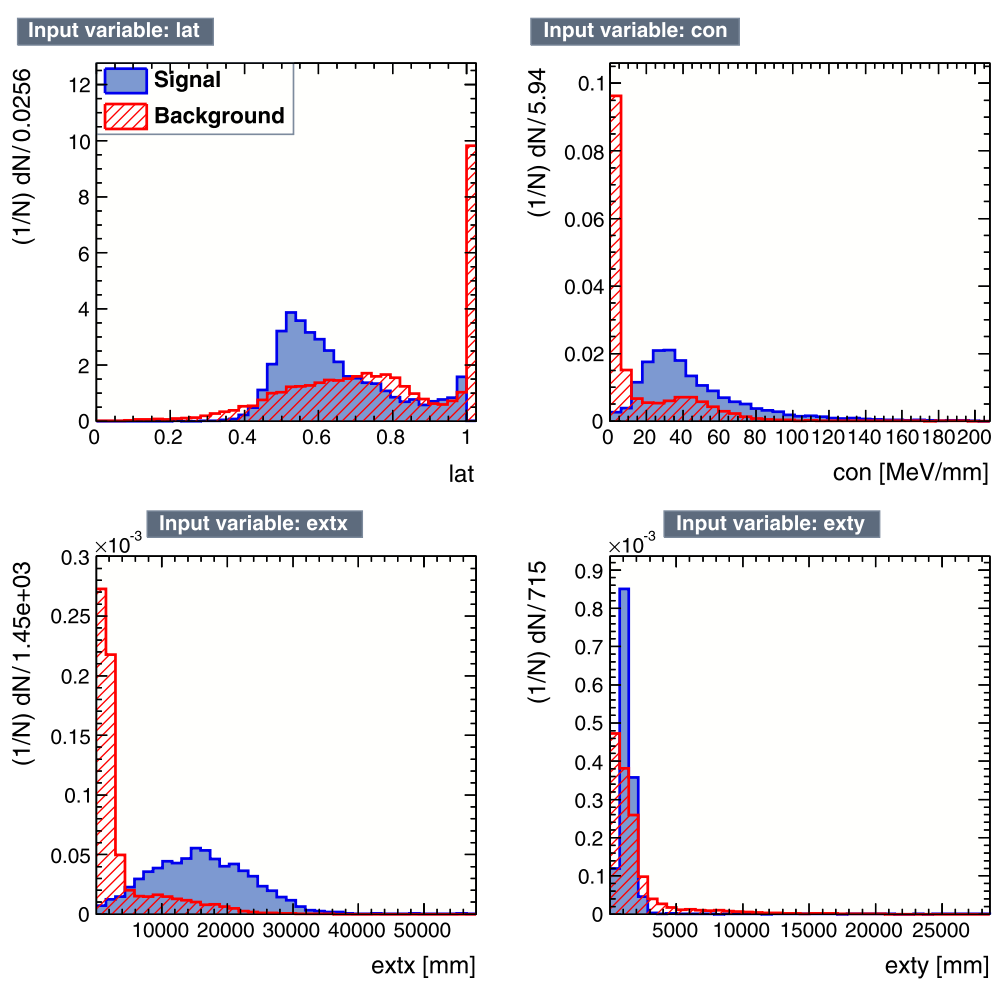

Similar robustness should be pointed out with respect to hit smearing effects, i.e. the case when individual hits are represented by more realistic volumes of three to six $\mathrm{mm}$ cubes (or even asymmetric dimensions). Our proposed variables are purely measures of the geometry of structures in a LArTPC. As long as the smallest spatial measure, i.e. a hit, is small compared with the extent of shower/track structures, we do not expect any of our analysis results to change significantly.

\section{Conclusion}

We have presented a study identifying particles in event structures obtained in high-granularity detectors such as a liquid argon TPC using four discriminating variables. Combining them using MVA algorithms from the TMVA toolkit gives signal efficiencies for Electron and Muon identification well above $90 \%$ with high background rejection efficiencies on all Neutrino interaction channels. Protons, Kaons and charged Pions show anything from acceptable to poor background rejection but high signal efficiency. Neutral Pion identification can be made very efficient if a background contamination of around $16 \%$ is not a concern. These results depend on the two specific case studies undertaken here: first for a possible neutrino beam from CERN to Finland and second, for a similar neutrino beam (same flavour weighting factors) but uniform neutrino energy distribution.
The data used in this work has been assumed to consist of hits containing three spatial coordinate values and one energy value, which should be the case for LAr TPC detectors. Furthermore, it is assumed that each single structure to be analysed has been clustered correctly which is still an outstanding challenge for automated data analysis on high granularity detector data. Nevertheless, if such a clustering can be achieved by some means, then this work proposes an efficient and reliable method to identify Electron (as well as Positron) events in a combined hadronic and leptonic background. These would be the primary signatures of charged current Electron-Neutrino interactions which constitute the main target of future long baseline experiments. It also allows the identification of neutral Pions without additional knowledge of decay vertices (or similar quantities) in a mixed background of other Hadrons and Leptons, and can be used for Muon identification and as an efficient first pass on Proton, Kaon and charged Pion identification.

Acknowledgements We acknowledge the contribution made to this study by Timothy Hughes and Laurence Woodward who worked with the corresponding author as final year project students on this topic. Support on the STFC consolidated grant ST/H00369X/1 is also acknowledged.

Open Access This article is distributed under the terms of the Creative Commons Attribution License which permits any use, distribution, and reproduction in any medium, provided the original author(s) and the source are credited. 


\section{References}

1. A. Rubbia, J. Phys. Conf. Ser. 308, 012030 (2011)

2. B. Fleming, J. Phys. Conf. Ser. 308, 012007 (2011)

3. M. Tanaka, J. Phys. Conf. Ser. 308, 012022 (2011)

4. C. Rubbia et al., J. Instrum. 6, P07011 (2011)

5. A. Ereditato, A. Rubbia, Nucl. Phys. B, Proc. Suppl. 154, 163 (2006)

6. E.F. Martinez, T. Li, S. Pascoli, O. Mena, Phys. Rev. D 81, 073010 (2010)

7. A. Longhin, PoS (ICHEP 2010) 325

8. C. Andreopoulos, Nucl. Instrum. Methods Phys. Res., Sect. A 614, 87 (2010)

9. S. Agostinelli et al., Nucl. Instrum. Methods Phys. Res., Sect. A 506, 250 (2003)

10. S. Amoruso et al., Nucl. Instrum. Methods Phys. Res., Sect. A 523, 275 (2004)
11. P. Speckmayer, A. Hoecker, J. Stelzer, H. Voss, J. Phys. Conf. Ser. 219, 032057 (2010)

12. I.T. Jolliffe, Principal Component Analysis, 2nd edn. (Springer, New York, 2002)

13. B. Andrieu et al., Nucl. Instrum. Methods Phys. Res., Sect. A 344, 492 (1994)

14. E. Aprile, A.E. Bolotnikov, A.I. Bolozdynya, T. Doke, Noble Gas Detectors (Wiley-VCH, Weinheim, 2006)

15. P. Huber, M. Lindner, W. Winter, Comput. Phys. Commun. 167, 195 (2005)

16. C.B. Barber, D.P. Dobkin, H.T. Huhdanpaa, ACM Trans. Math. Softw. 22, 469 (1996)

17. T.E. Oliphant, Comput. Sci. Eng. 9, 10 (2007)

18. A. Ankowski, et al. (ICARUS coll.), Acta Phys. Pol. B 41, 103 (2010) 